\title{
Patterns of Pediatric Malaria Incidence and Outcomes in Kasungu, Malawi in 2016
}

\author{
Byungchan Kim1, Sohyun Chang', Min Jae Shin'2, Prescott Hong ${ }^{3}$, George Lee 3 , Min Jung Koh ${ }^{3}$, \\ Immanuel H. Anaborne ${ }^{3}$, Donghoon Lee ${ }^{3}$, Paul S. Chung ${ }^{3}$, George Talama ${ }^{4}$, Thomas Nyirenda ${ }^{5}$
}

\author{
${ }^{1}$ Department of Radiology, Washington University, St. Louis, MO, USA \\ ${ }^{2}$ Department of Art \& Science, Biology, Johns Hopkins University, Baltimore, MD, USA \\ ${ }^{3}$ Youth with Talents, Fairfax, VA, USA \\ ${ }^{4}$ Kasungu District Hospital, Kasungu District, Malawi \\ ${ }^{5}$ Clinical Research Education and Management Service, Lilongwe, Malawi \\ Email: paulschung08@gmail.com
}

How to cite this paper: Kim, B., Chang, S., Shin, M.J., Hong, P., Lee, G., Koh, M.J., Anaborne, I.H., Lee, D., Chung, P.S., Talama, G. and Nyirenda, T. (2019) Patterns of Pediatric Malaria Incidence and Outcomes in Kasungu, Malawi in 2016. Advances in Infectious Diseases, 9, 277-284.

https://doi.org/10.4236/aid.2019.94021

Received: September 20, 2019

Accepted: November 23, 2019

Published: November 26, 2019

Copyright $\odot 2019$ by author(s) and Scientific Research Publishing Inc. This work is licensed under the Creative Commons Attribution International License (CC BY 4.0).

http://creativecommons.org/licenses/by/4.0/

cc) (i) Open Access

\begin{abstract}
Malawi is resource poor country in Africa with one of the highest infant and child mortality rates in the world. Malaria is one of the major causes of morbidity and mortality among children in Malawi. Studies have shown barriers to care of sick children and the formal health sector has shown sub-optimal patient assessments and overreliance on antibiotic treatment of a child with fever. In 2017, a team of students from Korea and USA audited clinic registers of six health centres under the hospital's administration of Kasungu District in Malawi for the year of 2016. The clinics were included in the study were those that contributed to the largest number of patients in the district annually. The aim of the audit was to examine treatment outcomes of all children presenting with fever and on whom a definitive diagnosis or presumptive diagnosis of malaria was made at the 6 chosen clinics in Kasungu for improvement of health information system for the district health office. The number of children with malaria ranged between 80 and 440 per month, with the peak occurring between the rainy months of February to May. Peak mortality rate occurred in September and November at 21\%. Patients presented with multiple symptoms of fever, convulsion, pallor, vomiting, cough, diarrhea, abdominal pain and headache. Average mortality rate differed between traditional authorities, ranging from $4 \%$ to $15 \%$. Off-season high mortality finding promotes more studies looking into socioeconomic and hospital support. Multiple symptoms among patients treated for malaria is a common finding emphasizing the need to integrate household practices for malaria prevention, good malaria case management and linkage with community care of childhood illness (CCM) programs at the clinics in the district.
\end{abstract}




\section{Keywords}

Case Management, Childhood Fever, Kasungu, Malawi, Mortality, Pediatric Malaria

\section{Introduction}

Malawi is a resource poor country with one of the highest neonatal and infant mortality rates in the world. In 2004, a Demographic and Health Survey report showed infant mortality ratio (IMR) of 76 per 1000 live births and a neonatal mortality ratio (NMR) of 27 per 1000 [1].

A similar survey done in 2010 reported that Malaria is endemic throughout Malawi and it is the leading cause of morbidity and mortality in children under age 5 and pregnant women [2]. The report further stated that ninety-eight percent of malaria infections in Malawi are caused by Plasmodium falciparumwith Anopheles funestus, A. gambiae, and A. arabiensis as the primary mosquito vectors. Malaria transmission is largely determined by climatic factors, including temperature, humidity, and rainfall. Vector abundance follows seasonal rainfall patterns, and an increase in temperature raises the parasite's reproductive rate, thereby influencing the prevalence rate of malaria in the population. Transmission is higher in areas with high temperatures and during the rainy season (October through April), particularly along the lakeshore and lowland areas of the lower Shire Valley, the report concluded.

Health care to children is not equitable in Malawi. Studies have shown that children from the poorest households and children aged more than 12 months were less likely to seek care when compared to those living in wealthier households and children of higher age-group respectively. These studies have also shown that families from rural households spent more time travelling to a health facility compared to urban households and that, visiting a trained healthcare provider was associated with longer travel time and higher direct costs compared to visiting an untrained provider [3].

Due to a weak public health system, Malawi has implemented community care of childhood illness (CCM) program. Recommended by the World Health Organization, CCM program integrates contributions of community health workers in managing childhood diseases including malaria, pneumonia, and neonatal sepsis [3] [4]. The CCM program has shown to be a promising strategy for increasing coverage of sick child treatment, even with room for improvement, especially in the correct assessment signs [5].

However, integrated pediatric fever management in the country has been shown to be sub-optimal for completed assessments and antibiotic targeting despite common compliance to malaria treatment guidelines, with malaria rapid test negative results being strongly associated with antibiotic over-treatment conditioned by cough or difficult breathing complaints [6]. 
Our team looked at the burden and treatment outcomes of children both aged under five and over five who presented to five health facilities and the Kasungu District Hospital of Kasungu, a rural district in central Malawi. The region is the home to 843,000 of 17.5 million people in Malawi [7]. Previous mapping of malaria transmission in Malawi has shown that the Kasungu region have been severely affected, with evidence of increased prevalence in 2010 [8].

The aim of the audit was to examine treatment outcomes of all children presenting with fever for whom a definitive diagnosis or presumptive diagnosis of malaria was made at the six chosen clinics in Kasungu to facilitate public health response to pediatric malaria.

\section{Methods}

In 2017, a team of students from Korea and USA obtained authority from the District Health Officer of Kasungu District hospital to digitalize clinic registers of six health centres under the hospital's administration of Kasungu for the year of 2016. Selection criteria were all pediatric patients (aged 18 or less) admitted to the hospital in the year of 2016 were considered in this study. Clinics were chosen by the number of annual patient visits to each clinic. The students were given access to out-patient registers to extract data on all children presenting with fever and on whom a definitive diagnosis or presumptive diagnosis of malaria was made. The extracted data included region of residence, age, symptoms, diagnosis, length of stay, and outcomes. Patients were divided between two age groups: under 5 years and over 5 years.

The extracted data was analyzed for trends between age groups, months of visit, and regions of residence. Ethics approval from the National Health Research Committee was not requested as this was an audit from registers and did not involve handling of human subjects or their stored samples.

\section{Results}

The number of pediatric patients with malaria ranged between 80 and 500 per month (Figure 1(a)), amounting to 2390 diagnoses in the study year. The number of cases was steady at around 100 patients a month between August and December. Meanwhile, the monthly incidence rose from January to April, before decreasing from May to July, in consistence with the pattern described in the National Demographic and Health Surveys of Malawi. At peak times, 440 patients were admitted. Similar trend was observed for the number of deceased patients per month, with peak number of 32 deaths in April (Figure 1(b)). From January to April, the number of children dying among those that were registered rose from 10 to 35 per month. Mortality rate showed consistent rate of 5\% from January to August and December (Figure $1(\mathrm{c})$ ). Mortality rate fluctuated with increases in September and November, reaching $21 \%$ mortality rate in November.

Approximately $70 \%$ of the patients were aged under 5. Across both age groups, the most common presented symptoms were fever, convulsion, pallor, 


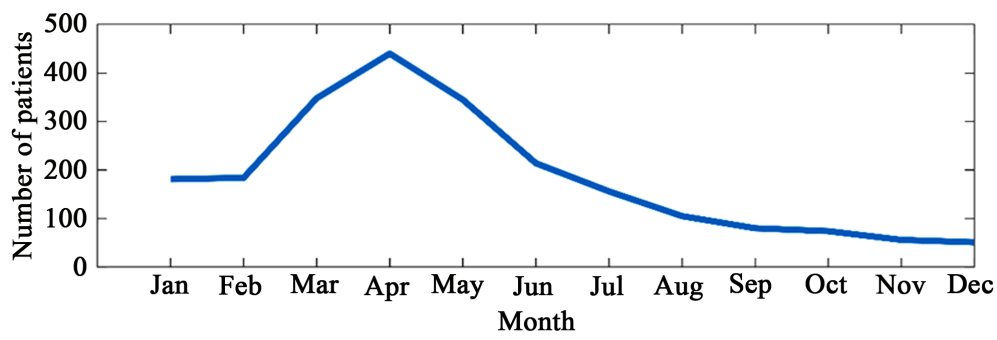

(a)

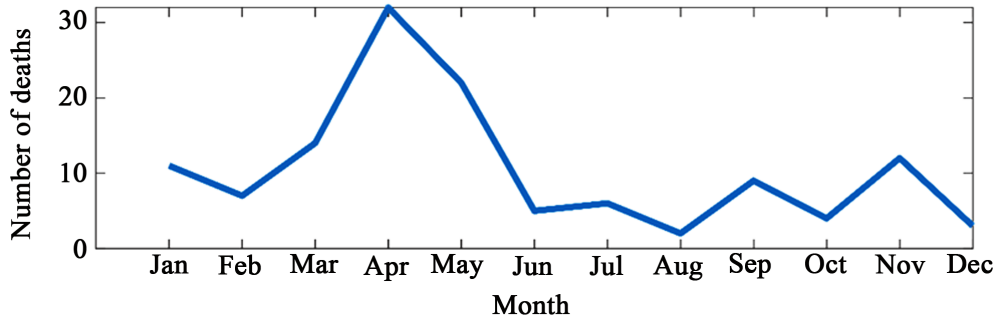

(b)

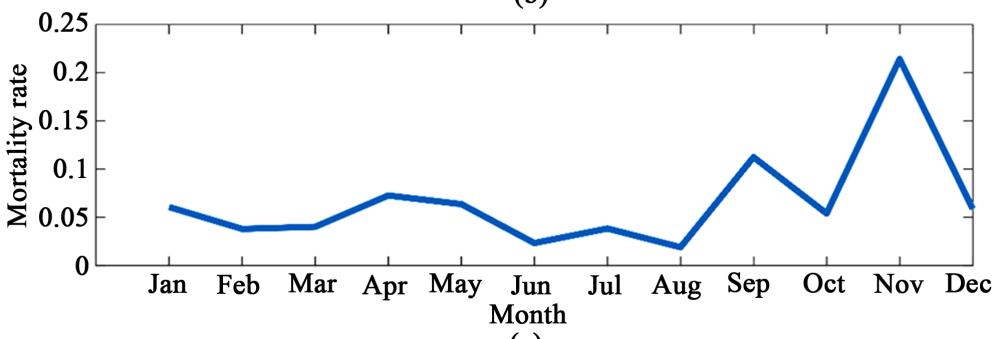

(c)

Figure 1. 2016 monthly trend of Kasungu region hospitalization and deaths due to malaria in children. (a) Number of pediatric patients admitted to hospital for overnight care per month; (b) Number of pediatric deaths at the hospital per month; (c) Mortality rate of pediatric patients admitted.

vomiting, cough, diarrhea, abdominal pain and headache in descending prevalence order (Figure 2(a)). Patients over 5 years of age did not present convulsions, coughs and diarrhea as often, but showed abdominal pain and headache more commonly. Mortality rate amongst pediatric patients greater than 5 years of age was higher, although statistical significance could not be inferred (Figure 2(b)). There were no significant differences in length of stay after admission (Figure 2(c)).

To observe changes in pediatric malaria incidence and outcomes in more spatial detail, the patient data were sorted by their authorities of residence. The Kasungu region is divided into numerous traditional authorities, of which the six authorities with largest pediatric malaria population were studied. The six authorities accounted for $31.4 \%$ of all admitted patients (702 out of 2238). Largest patient population came from Kaomba at 321 pediatric Malaria patients, followed by Mwase at 123 patients (Figure 3(b)). In all six traditional authorities analyzed, fever was the most common symptom followed by convulsion (Figure 3(a)). Mortality rate differed between traditional authorities. Pediatric patients from Mwase traditional authority showed the lowest mortality rate at $4 \%$ while patients from Chulu showed the highest mortality rate at 15\% (Figure 3(c)). 


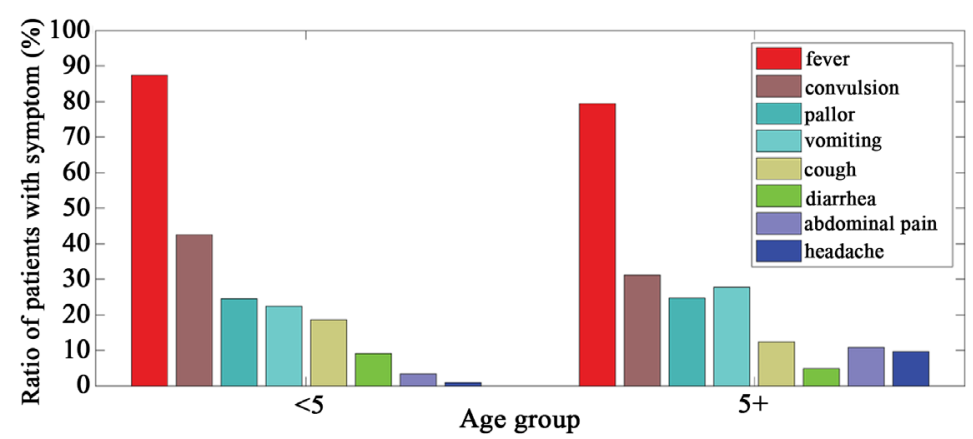

(a)

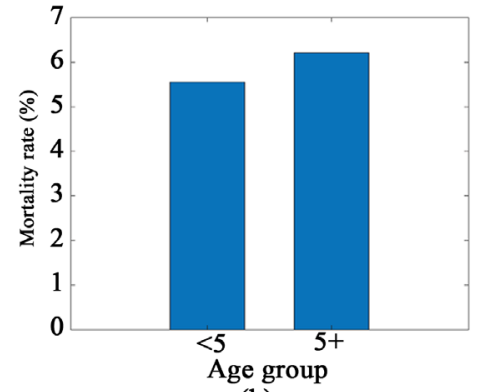

(b)

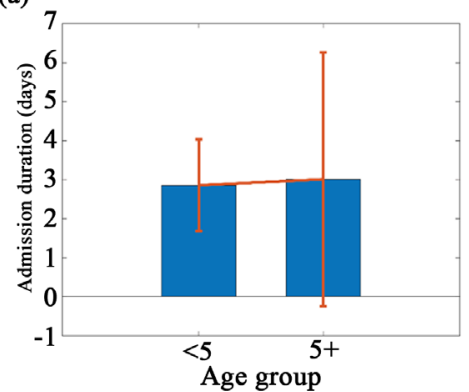

(c)

Figure 2. Age effects on inpatient malaria outcomes. (a) The ratio of occurrence of common malaria symptoms in children under and over age of 5; (b) Mortality rate of children admitted to hospital for malaria categorized by age; (c) Average duration of admission categorized by age groups. Error bars show standard deviation of duration of admission amongst the age groups.
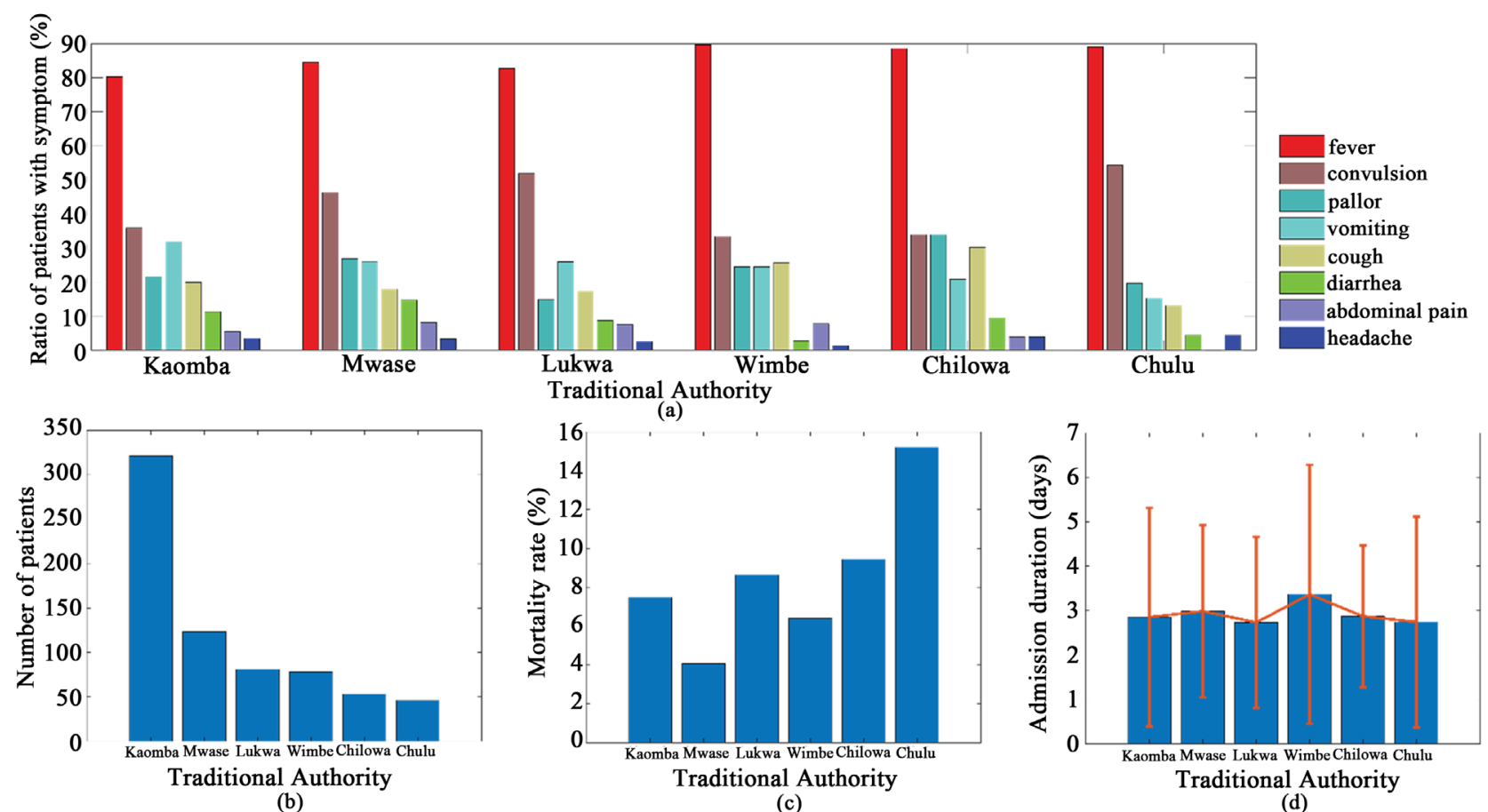

Figure 3. Inpatient pediatric malaria outcomes in populous traditional authorities. (a) The ratio of patients' symptom for the six traditional authorities with the greatest number of patients admitted; (b) Number of pediatric patients admitted to the hospital for each traditional authority; (c) Mortality rate for each traditional authority; (d) Admission duration of average pediatric malaria patient for each traditional authority. Standard deviation of admission duration is piloted as the error bars. 
Admission duration averaged around 3 days for all traditional authorities, with no significant difference between the districts (Figure 3(d)).

\section{Discussion}

Analysis of monthly diagnoses and admission trend has shown that malaria incidence in Kasungu district of Malawi is concentrated on the months of January to April. This picture is consistent with that described in Malawi Demographic and Health Surveys which provides crude data for Malawi but not disaggregated by district. Our study shows that in Kasungu district of Malawi malaria cases are high among children in the period of January to April which coincides with rainy season when mosquitoes breed in large numbers. Thus, it may be inferred that the rise in number of mosquitoes cause the high malaria incidence rate during those months. This shows that despite many efforts to educate the people about prevention of mosquito bites more is required.

The number of pediatric deaths caused by malaria also rose during the same months of January to April, although the mortality rate remained stable at $5 \%$. Multiple factors could cause this stable pattern in mortality rate. Parents may be more cognizant of potential malaria cases during these months, shortening the time between incidence and admission to hospital where the pediatric patients can obtain professional medical care. An increased influx of medical supplies for malaria during those months could lead to consistent quality of healthcare despite increased number of patients. Good practices associated with this stability need to be encouraged among medical staff and communities. Mortality peaked in September and November reaching up to $20 \%$. This unacceptably high mortality rate may be due to lack of supplies and delay in recognition of malaria. More health promotion against such high mortality is required.

Our data also shows that treating a child with fever that could be due to Malaria in Kasungu is complex. Disease presentation was complicated amongst children with multiple symptoms like fever, convulsions, pallor, vomiting, cough, diarrhea, abdominal pain and headache. The complexity of additional diseases in the patients treated for malaria which may be related to Malaria or not requires advanced clinical and nursing skills as well as strong health worker skills for integrated disease management in children. Our study did not explore how many clinic staff possess these advanced skills but the finding points to a strong need to have skilled workers attending to sick children in the district.

Our audit also shows that malaria even in a small geographical area of Kasungu with 7878 square kilometers in size mortality rate differs substantially between traditional authorities. This finding may signify the inequalities in preventive healthcare and treatments provided in each region of the district. But there are other contributing factors that need more studies to be understood. What our study showed is that a large proportion of childhood patients with malaria in Kasungu district come from the rural parts of the district. Unfortunately, the discrepancy in preventive care for malaria found by other prior stu- 
dies [9] in Malawi cannot explain the same discrepancy in malaria incidence between regions in Kasungu that we observed.

\section{Conclusions and Limitations}

Our study showed that malaria among children in Kasungu District of Malawi is highly prevalent, seasonal, complicated with other diseases in the same patient and associated with high mortality. This is consistent with the National Demographic and Health Surveys of Malawi that does not focus on Kasungu district per se. Several limitations exist in this study. Firstly, this audit does not tell us the proportion of malaria cases don't make it to the selected clinics. So, the burden of disease and mortality could be higher that our data shows. Secondly, handling such a complex disease in a vulnerable population requires good supply of drugs, equipment and skilled staff. Our study did not correlate the burden of disease with available resources therefore we are unable to explain beyond observable difference by seasons and regions of the district. In addition, quantified data for quality of life in each traditional authority of the district is not available, leading to lack of detailed analysis on the effect of such factors in the differences of malaria incidence and mortality rate observed in the district.

Still, we believe that this audit provides certain insights in terms of the trends in malaria incidence and the high mortality rates in all seasons. The findings of our audit show that pediatric malaria is highly prevalent and emphasizes the need to integrate household practices for malaria prevention, good malaria case management and linkage with community care of childhood illness (CCM) programs at the clinics in Kasungu. However, from this study we cannot ascertain how well covered the district is with CCM which is very beneficial to Malaria control.

\section{Acknowledgements}

The authors acknowledge the HeMemics Biotechnologies, Inc, and Youth with Talents for sponsoring this medical project (HB2018-06PH). We would like to thank staff from Kasungu District Hospital for their advice and assistance throughout this study. Furthermore, we are thankful for the permission and opportunity to conduct a retrospective analysis on their hospital data. The authors would like to thank Clinical Research Education and Management Services Ltd of Lilongwe who contributed to the editing of this paper.

\section{Conflicts of Interest}

The authors declare no conflicts of interest regarding the publication of this paper.

\section{References}

[1] National Statistical Office (NSO) [Malawi] and ICF Macro (2004) Malawi Demographic and Health Survey 2004: Preliminary Report. NSO and ORC Macro, Cal- 
verton. https://www.dhsprogram.com/pubs/pdf/FR175/FR-175-MW04.pdf

[2] National Statistical Office (NSO) [Malawi] and ICF Macro (2011) Malawi Demographic and Health Survey 2010: Preliminary Report. NSO and ORC Macro, Calverton. https://dhsprogram.com/pubs/pdf/FR247/FR247.pdf

[3] Ustrup, M., Ngwira, B., Stockman, L.J., et al. (2014) Potential Barriers to Healthcare in Malawi for Under-Five Children with Cough and Fever: A National Household Survey. Journal of Health, Population and Nutrition, 32, 68-78.

https://www.ncbi.nlm.nih.gov/pmc/articles/PMC4089074/

[4] Haines, A., Sanders, D., Lehmann, U., et al. (2007) Achieving Child Survival Goals: Potential Contribution of Community Health Workers. Lancet, 369, 2121-2131. https://doi.org/10.1016/S0140-6736(07)60325-0

[5] Gilroy, K.E., Callaghan-Koru, J.A., Cardemil, C.V., et al. (2013) Quality of Sick Child Care Delivered by Health Surveillance Assistants in Malawi. Health Policy Plan, 28, 573-585. https://doi.org/10.1093/heapol/czs095

[6] Johansson, E.W., Selling, K.E., Nsona, H., et al. (2016) Integrated Paediatric Fever Management and Antibiotic Over-Treatment in Malawi Health Facilities: Data Mining a National Facility Census. Malaria Journal, 15, 1-12.

https://doi.org/10.1186/s12936-016-1439-7

[7] (2018) 2018 Population and Housing Census: Preliminary Report. https://malawi.unfpa.org/sites/default/files/resource-pdf/2018\%20Census\%20Preli minary\%20Report.pdf

[8] Bennett, A., Kazembe, L., Mathanga, D.P., et al. (2013) Mapping Malaria Transmission Intensity in Malawi, 2000-2010. The American Journal of Tropical Medicine and Hygiene, 89, 840-849. https://doi.org/10.4269/ajtmh.13-0028

[9] Holtz, T.H., Marum, L.H., Mkandala, C., et al. (2002) Insecticide-Treated Bednet Use, Anaemia, and Malaria Parasitaemia in Blantyre District, Malawi. Tropical Medicine \& International Health, 7, 220-230. https://doi.org/10.1046/j.1365-3156.2002.00846.x 